\title{
Todes-Anzeige.
}

Am I 4. Mai d. J. verschied nach kurzer Krankheit der Akademiker Theodor Bredichin. Mit ihm verlor Rußland seinen hervorragendsten Astronomen, der nicht nur durch wissenschaftliche Arbeiten, sondern auch als Professor und Direktor der beiden größten russischen Sternwarten - Moskau und nachher Pulkowo einen großen Einfluß auf den Entwickelungsgang der Astronomie hierzulande ausgeübt hat. Als Professor verstand er in hohem Grade die lernende Jugend für die Astronomie zu interessieren und zwar dauernd; nicht nur momentan durch zundende Rednergabe; er konnte auf eine stattliche Reihe Schüler hinweisen, die jetzt zu den leitenden Astronomen Rußlands zählen.

Bredichin wurde $183 \mathrm{I}$ den 8 . Dezember in Nicolajeff geboren und stammte aus einem alten Adelsgeschlechte. An dem bekannten Richelieuschen Lyzeum in Odessa erhielt er eine gründliche klassische Bildung und wurde $185 \mathrm{I}$ als Student der physico-mathematischen Fakultät der Universität in Moskau immatrikuliert. In Jahre 1857 wurde er zum Professor der Astronomie und 1873 außerdem zum Direktor der Sternwarte dieser Universität ernannt. Als solcher entfaltete er eine vielseitige Tätigkeit. Astrospektroskopische Beobachtungen - die ersten in Rußland -, Ortsbestimmungen von Sternen, Schwerebestimmungen durch Pendelbeobachtungen wurden ausgeführt, seine Hauptarbeiten aber waren die bekannten Untersuchungen über die Kometenformen und im Zusammenhang damit seine Theorie der Meteore. Im Jahre 1890 folgte Bredichin dem Rufe an die Kaiserliche Akademie der Wissenschaften und wurde Nachfolger O. Struves als Direktor der Nicolai-Hauptsternwarte. Während dieser Zeit ist unter anderen Neuerungen auch die Anschaffung eines astrophotographischen Refraktors anzuführen, wodurch die Himmelsphotographie in das Programm der Tätigkeit der Sternwarte tatsächlich eingereiht wurde. Wegen angegriffener Gesundheit und um sich ungestört seiner Lebensaufgabe, der Theorie der Kometen, widmen zu können, gab er Ende 1894 seine Stellung als Direktor auf und lebte als Akademiker in Petersburg, wo er sich bis zuletzt mit wissenschaftlichen Arbeiten beschäftigte.

Noch kurz vor seinem Tode veröffentlichte er eine Zusammenstellung seiner Hauptarbeiten auf dem Gebiete der Kometentheorie. Etwa 150 größere und kleinere Arbeiten, von denen die meisten dieses Gebiet berühren, sind aus seiner Feder hervorgegangen.

Bredichin war eine begabte und vielseitig gebildete Persönlichkeit; seine etwas nervöse, aber impulsive Natur war den edelsten Regungen zugänglich, die dank seiner unabhängigen materiellen Lage in vielen und weitgehenden Hinsichten praktisch sich betätigten. Davon legen seine Stiftungen von Prämien für wissenschaftliche Arbeiten und die vielfache Förderung einzelner Unternehmungen durch pekuniäre Unterstiltzung Zeugnis ab.

Pulkowo, den 9. Juni 1904.

O. Backlund.

\section{Ephemeride des Planeten (217) Eudora.}

Zur Verfolgung des in Heidelberg photographierten, seit I890 nicht beobachteten Planeten (2I 7) Eudora ist folgende Ephemeride für I $2^{\text {h }}$ M. Z. Berlin gerechnet:

\begin{tabular}{|c|c|c|c|c|c|}
\hline \multicolumn{2}{|c|}{1904} & $\alpha$ & $\delta$ & $\log r$ & $\log \Delta$ \\
\hline Juni & I 7 & I $7^{h} 54^{m} 32^{s}$ & $-4^{\circ} 5^{2 !} \cdot 5$ & & \\
\hline & I 9 & $52 \quad 55$ & 452.9 & 0.335 & 0.070 \\
\hline & $2 \mathrm{I}$ & 5 I 18 & $4 \quad 54.2$ & & \\
\hline & 23 & 4942 & $4 \quad 56.3$ & & \\
\hline & 25 & $4^{8} \quad 6$ & $4 \quad 59.4$ & & \\
\hline & 27 & 4631 & $53 \cdot 5$ & 0.331 & 0.065 \\
\hline & 29 & I7 4457 & -5 & & \\
\hline
\end{tabular}

\begin{tabular}{|c|c|c|c|c|}
\hline 1904 & $\alpha$ & $\delta$ & $\log r$ & $\log A$ \\
\hline Juli & $I 7^{\mathrm{h}} 43^{\mathrm{m}} 25^{\mathrm{s}}$ & $-5^{\circ}$ I $4^{\prime} 7$ & & \\
\hline 3 & $4 I \quad 57$ & 521.6 & & \\
\hline 5 & 4033 & $\begin{array}{ll}5 & 29.4\end{array}$ & 0.328 & 0.066 \\
\hline 7 & $39 \times 3$ & 538.0 & & \\
\hline 9 & $37 \quad 57$ & $\begin{array}{ll}5 & 47.4\end{array}$ & & \\
\hline I I & $3^{6} 47$ & $5 \quad 57.6$ & & \\
\hline I 3 & I 7354 I & $\begin{array}{ll}-6 & 8.4\end{array}$ & 0.324 & 0.072 \\
\hline
\end{tabular}

Gr. I I.5. Korrektion der Ephemeride Heidelberg Juni $I_{4}:+0{ }^{\mathrm{m}} 7+\mathrm{I}^{\prime}$.

Berlin, I904 Juni I 6.

P. V. Neugebauer.

(419) Anrelia. Korrektion der Ephemeride (Veröff. R.I. 22): Juni I $3-5^{\text {s }} 0^{\circ}$. $\mathcal{F}$. Palisa.

\section{Inhalt:}

Zu Nr. 3958. M. Luizet. Observations d'étoiles variables à periode longue ou inconnue. 337. - E. Schaer. Une modification du réflecteur Newtonien. 345. - L. Gabba. Osservazioni di pianetini e cometa. 347. - M. Wolf. Beobachtangen von kleinen Planeten. 349. - A. Berberich. Planet (149) Medusa. 349. - O. Backlund. Anzejge des Todes von Theodor Bredichin. 351. - P.V. Neugebauer. Ephemeride des Planeten (217) Eudora. 351. - F. Palisa. (419) Aurelia. 35 i. 